\title{
Acute myeloid leukemia with monosomy 7, ectopic virus integration site-1 overexpression and central diabetes insipidus: A case report
}

\author{
HONGBING MA, JING YANG, BING XIANG and YONGQIAN JIA \\ Department of Hematology, West China Hospital, Sichuan University, Chengdu, Sichuan 610041, P.R. China
}

Received July 2, 2014; Accepted February 5, 2015

DOI: $10.3892 / \mathrm{ol} .2015 .3139$

\begin{abstract}
Central diabetes insipidus (DI) is a rare complication in patients with acute myeloid leukemia (AML), typically occurring in patients with abnormalities of chromosomes 3 or 7. The association between AML with monosomy 7 and DI has been described in a number of studies; however, DI has been rarely reported in cases of ectopic virus integration site-1 (EVII)-positive AML with monosomy 7. The current study reports a case of AML with monosomy 7 and EVI1 overexpression, with central DI as the initial symptom. The patient was an 18-year-old female who presented with polyuria and polydipsia. Bone marrow aspiration revealed $83.5 \%$ myeloperoxidase-positive blasts without trilineage myelodysplasia. The karyotype was $45, \mathrm{XX},-7$, and the patient presented monosomy 7 and EVII overexpression $\left(-7 / E V I 1^{+}\right)$ without $3 q$ aberration. Treatment with induction therapy was unsuccessful. To the best of our knowledge, this is the second case of DI-AML with $-7 / E V I 1^{+}$and without a $3 q$ aberration. The possible mechanisms associated with EVII, monosomy 7 and DI were investigated.
\end{abstract}

\section{Introduction}

Acute myeloid leukemia (AML) represents a group of myeloid neoplasms characterized by the accumulation of myeloblasts in the bone marrow and/or blood (1). Central diabetes insipidus (DI) is a rare complication observed in $<0.6 \%$ of patients with AML $(2,3)$, which typically occurs in patients with abnormalities in chromosomes 3 or 7 (4-7). Central DI is a condition characterized by extreme thirst and excessive urination, that is caused by a deficiency of antidiuretic hormone $(\mathrm{ADH})(8)$. A number of case

Correspondence to: Dr Hongbing Ma, Department of Hematology, West China Hospital, Sichuan University, 37 Guoxue Alley, Chengdu, Sichuan 610041, P.R. China

E-mail: rogermahb@163.com

Key words: ectopic virus integration site-1, acute myeloid leukemia, diabetes insipidus, monosomy 7 studies have described an association between DI and AML with monosomy $7(4,6,7,9,10)$; however, DI has been rarely reported in cases of ectopic virus integration site-1 (EVII)-positive AML with monosomy 7. The EVIl gene is located on chromosome 3q26 and encodes a 1,051-amino acid DNA-binding phosphoprotein, which functions as a transcription factor $(11,12)$. Groschel et al $(13)$ reported the that EVII overexpression is observed in $10.7 \%$ AML patients. The present study reports the case of an 18-year-old female suffering from AML with monosomy 7 and EVII overexpression $\left(-7 / E V I 1^{+}\right)$without $3 \mathrm{q}$ aberration, who presented with central DI as the initial symptom. In addition, the present study investigated the possible mechanisms associated with EVII, monosomy 7 and DI.

\section{Case report}

In December 2012, an 18-year-old female presented at West China Hospital (Chengdu, China) with complaints of fatigue, ecchymosis, polyuria and polydipsia lasting for two months. A physical examination performed upon admission revealed dry skin, indicating dehydration. A complete blood count revealed a white cell count of $151.93 \times 10^{9} / 1$, with $84 \%$ peripheral blasts, a hemoglobin level of $80 \mathrm{~g} / 1$ and a platelet count of $139 \times 10^{9} / 1$. In addition, electrolyte tests identified a serum sodium level of $162.4 \mathrm{mmol} / \mathrm{l}$ (indicating the presence of hypernatremia), serum osmolality level of $342 \mathrm{mOsm} / \mathrm{kg}$ and urine osmolality level of $128 \mathrm{mOsm} / \mathrm{kg}$. Magnetic resonance imaging of the brain revealed mild thickening of the pituitary stalk (Fig. 1), while cerebrospinal analysis was negative for leukemia. Central DI was suspected, and the patient was administered desmopressin acetate tablets ( $2 \mathrm{mg}$; Minirin; Ferring International Center SA, Saint-Prex, Switzerland) orally, three times a day. The polyuria, polydipsia and dry skin resolved, with normalization of serum sodium and serum osmolality levels. Bone marrow smear tests indicated a morphological diagnosis of AML with $83.5 \%$ blasts (Fig. 2). Cytochemical staining was negative for peroxidase and positive for periodic acid-Schiff (Baso Diagnostics Inc., Zhuhai, Taiwan). Flow cytometric analysis revealed $88 \%$ blasts in the total nucleated cell population expressing cluster of differentiation (CD) 34, human leukocyte antigen (HLA)-DR, CD13, CD117 and CD123 (data not shown), and a diagnosis of AML with minimal differentiation [French-American-British 

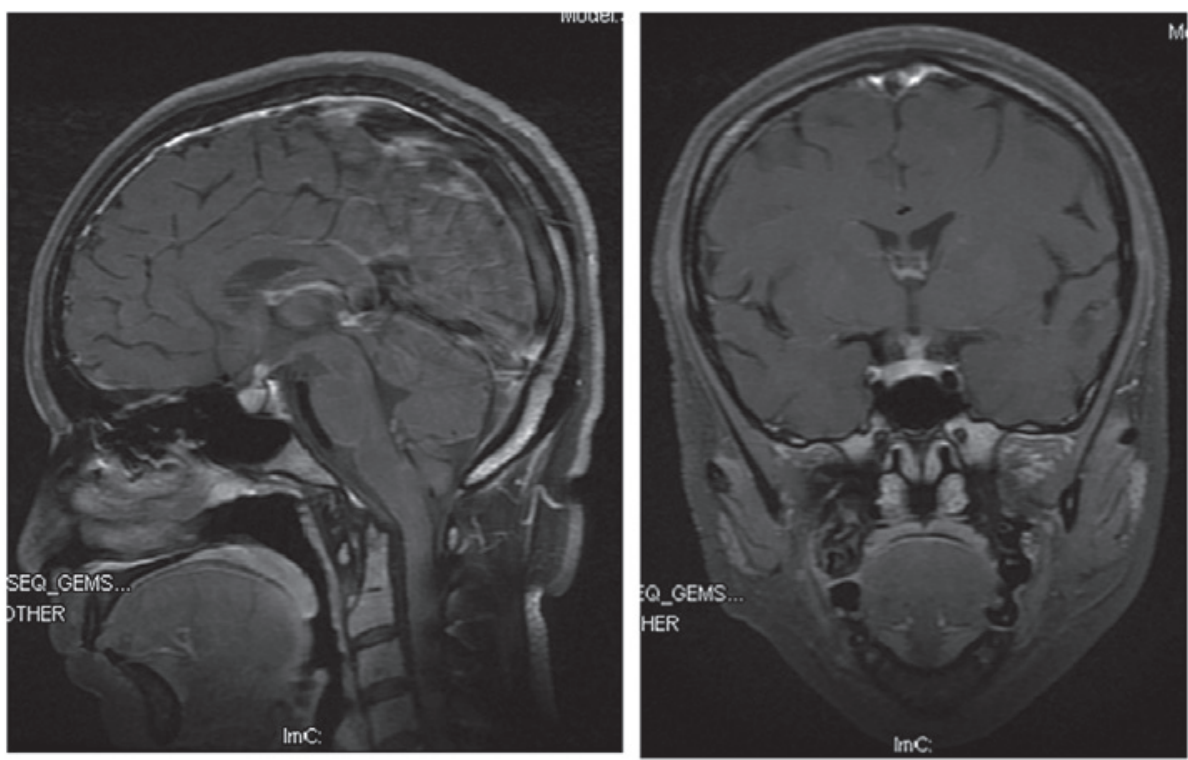

Figure 1. Magnetic resonance imaging of the brain (sagittal and coronal scans) revealed a thickened pituitary stalk.

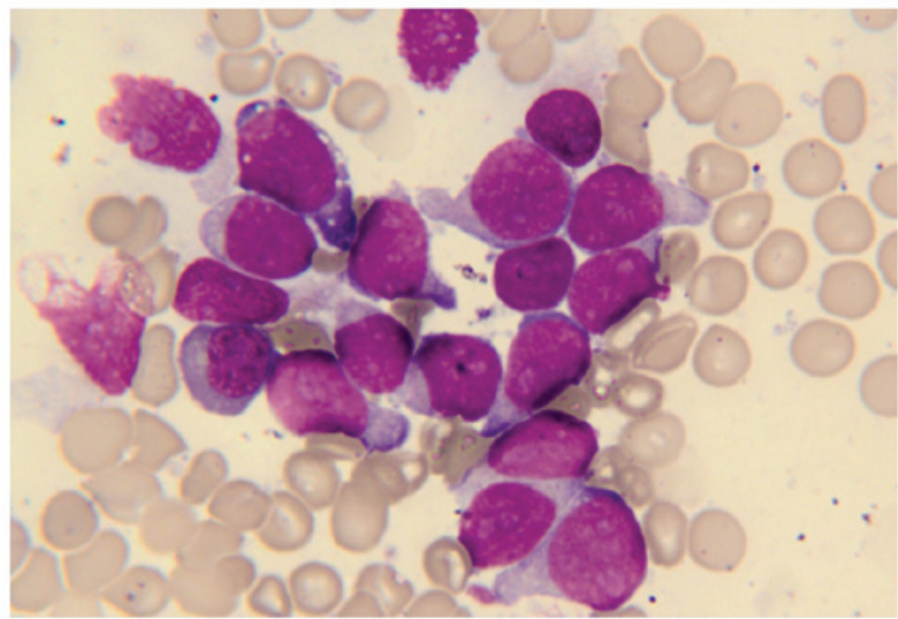

Figure 2. Bone marrow smear test revealed the presence of a high number of myeloblasts, which indicates the diagnosis of acute myeloid leukemia.

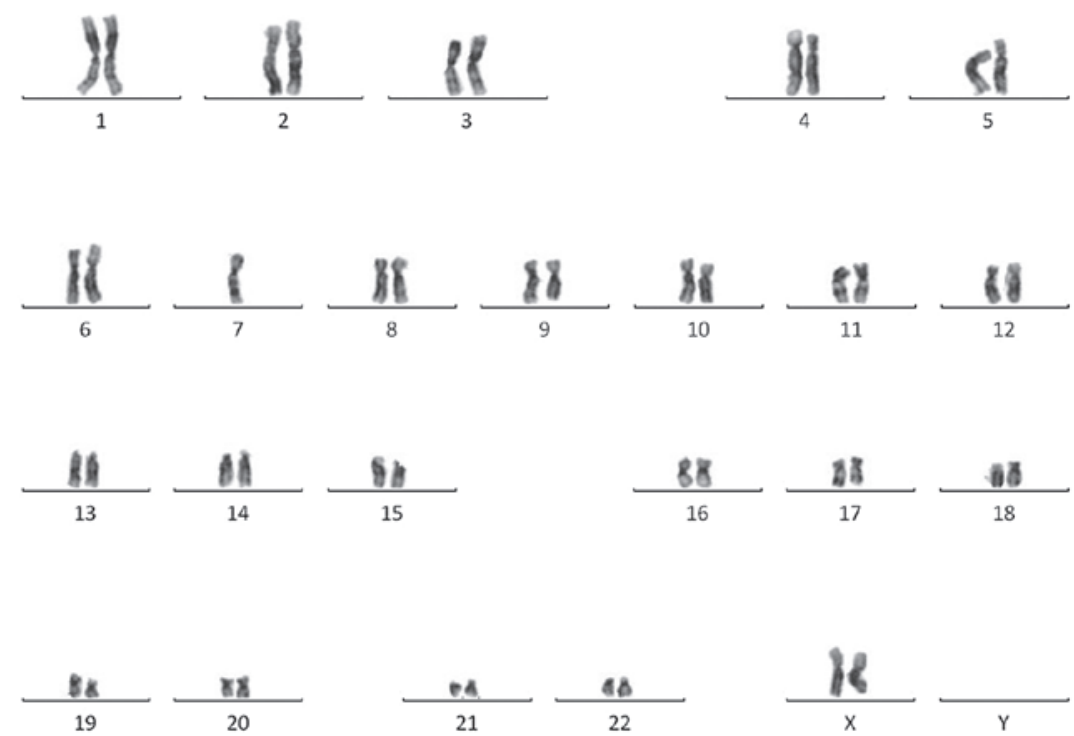

Figure 3. Karyotype of G-banded subclone with monosomy 7. 
classification, AML-M0 (14)] was determined. Trilineage dysplasia was unremarkable, while a cytogenetic analysis revealed a monosomy karyotype, 45,XX,-7 (Fig. 3). The patient was screened for fusion genes, revealing that EVII and T-cell leukemia homeobox 1 were positive, whereas FLT3/ITD, $C$-KIT D816V, nucleophosmin and CCAAT/enhancer binding protein $\alpha$ were negative. Furthermore, the patient had a family history of acute lymphocytic leukemia (pre-B), since the patient's father had been diagnosed with the disease at West China Hospital one year before the present diagnosis. Cytogenetic analysis and genetic screening of the bone marrow of the patient's father was performed to identify whether a common oncogene was present, however the results were unremarkable.

Subsequently, the patient was subjected to an initial cycle of induction chemotherapy with daunorubicin (45 mg/m², days 1-3) and cytarabine (200 mg/d, days 1-7); however, remission was not achieved. A second cycle of induction chemotherapy was administered using the FLAG regimen (50 mg fludarabine, days 1-5; 2 g cytarabine, days 1-5; $300 \mu \mathrm{g}$ granulocyte colony-stimulating factor, days 0-15) with no response. Treatment with arsenic trioxide (10 mg, days 1-21) and lenalidomide (10 mg, days 1-21) also produced no response. Without improvement or significant deterioration, the patient's platelet count remained high $\left(83 \times 10^{9} / 1-194 \times 10^{9} / 1\right)$ following chemotherapy. Due to the refractoriness to chemotherapy and the high risk associated with transplantation, the patient was reluctant to undergo allogeneic stem-cell transplantation, despite her sibling being an HLA match. Therefore, the patient continued to take desmopressin acetate tablets, and palliative care with low-dose cytarabine and supportive treatment were administered. However, the patient succumbed to a severe pulmonary infection 8 months after diagnosis.

\section{Discussion}

AML associated with DI (DI-AML) rarely occurs. The association between DI-AML and cytogenetic aberrations has been reported in a number of studies (1-3). The most common aberrations are monosomy 7 and $3 q$ alterations. Montecucco et al (15) and de la Chapelle et al (9) reported that $77 \%$ of DI-AML cases were associated with monosomy $7 q$ and $44 \%$ of cases were associated with $3 q$ alterations. In addition, Piccin et al (16) reviewed previously published reports of DI-AML, revealing that all 76 cases had acquired monosomy 7 . The finding of the aforementioned studies indicate that monosomy $7 q$ alterations may be a common 'chromosomal determinant' for DI-AML onset. DI-AML patients with $3 q$ aberrations have a number of common characteristics, including age (29-52 years), normal or high platelet count, hyperleukocytosis, trilineage myelodysplasia, no central nervous system involvement, failure to respond to first-line treatment or early relapse, and poor prognosis $(17,18)$. These features are termed 3q21q26 syndrome. The findings of the current study are consistent with such features, with the exception of trilineage myelodysplasia and $3 \mathrm{q}$ aberration. However, the patient also demonstrated EVII overexpression and monosomy-7 (-7/EVII $\left.{ }^{+}\right)$, consistent with one of the two cases reported by Piccin (15). To the best of our knowledge, this is the second case of DI-AML with $-7 / E V I 1^{+}$without a $3 q$ aberration.

The EVII gene is located on $3 \mathrm{q} 26$ and codes for a 1,051-amino acid DNA-binding phosphoprotein, which functions as a transcription factor $(11,12)$. Groschel et al (13) demonstrated that $\mathrm{EVII}^{+}$is associated with specific chromosome abnormalities, including inv(3)/t(3;3), monosomy 7 and 11q23 translocations. $E V I 1^{+}$was detected in $21 / 23$ AML patients with $\operatorname{inv}(3) / t(3 ; 3)$ and in 33/38 AML patients with monosomy 7 . The role of $E V I 1$ remains unclear; however, inappropriate $E V I 1$ activation, in combination with other undefined genetic alterations, are hypothesized to result in low levels of antidiuretic hormone (ADH) (18). As $~ 90 \%$ of circulating ADH is associated with platelets, it is postulated that platelet $\mathrm{ADH}$ originates in the hypothalamus and that chromosome 3 abnormalities are associated with dysthrombopoiesis, which may result in alterations in ADH levels or function $(7,19,20)$. However, this does not explain why DI-AML patients without $3 q$ alterations exhibit aberrations in ADH levels or function.

In the present study, the patient exhibited no cytogenetic evidence of chromosome 3 abnormalities. Curley et al (21) reported a case of DI-AML with $\mathrm{t}(3 ; 3)(\mathrm{q} 21 ; \mathrm{q} 26)$ and monosomy 7 that presented $E V I I$ overexpression at the onset; however, EVI1 overexpression was not detected upon AML relapse and DI did not recur. This indicates that EVII overexpression may be involved in the development of DI in AML patients. The prognosis of DI-AML is poor and patient survival, regardless of karyotype, is extremely low compared with a similar cohort of AML patients without DI. Monosomy 7 separates the disease into two entities (7). Patients with monosomy 7 tend to have a poorer complete remission (CR) rate and outcome compared with patients with other aberrations (7). Gröschel et al (13) reported that $31 / 33$ patients with $-7 / E V I 1^{+}$AML failed to achieve CR following the first induction therapy, and 31 patients succumbed to the disease after a median period of 8.6 months. All patients with $-7 / E V I 1^{+}$AML had a poor survival rate [two-year relapse-free survival (RFS), 0\%; two-year overall survival, 0\%]. Additionally, patients with $E V I 1^{+}$AML who received allogeneic stem cell transplantation during the first CR had significantly improved five-year RFS (33 $\pm 10 \%$ vs. 0\%) (13). The response to treatment and survival time in the present study are similar to the results of the study by Groschel et al (13).

In the current study, the case of an AML patient with monosomy 7 and EVII overexpression, who exhibited central DI as the initial symptom, was presented. Treatment with induction therapy was unsuccessful. This case suggests that the presence of $-7 / \mathrm{EVII}^{+}$may be involved in the development of DI-AML, which is a rare syndrome, and indicates an extremely poor prognosis. The association between EVII overexpression and poor prognosis of AML requires further investigation and thus, the establishment of aggressive treatment approaches, including stem cell transplantation, and novel clinical trials are required.

\section{Acknowledgements}

The study was supported by funding provided by the Office of Science and Technology in Sichuan Province (grant no. 2014SZ0006-6).

\section{References}

1. Hasserjian RP: Acute myeloid leukemia: advances in diagnosis and classification. Int J Lab Hematol 35: 358-366, 2013. 
2. Kimmel DW and O'Neill BP: Systemic cancer presenting as diabetes insipidus. Clinical and radiographic features of 11 patients with a review of metastatic-induced diabetes insipidus. Cancer 52: 2355-2358, 1983

3. Maghnie M, Cosi G, Genovese E, et al: Central diabetes insipidus in children and young adults. N Engl J Med 343: 998-1007, 2000.

4. Castagnola C, Morra E, Bernasconi P, Astori C, Santagostino A and Bernasconi $\mathrm{C}$ : Acute myeloid leukemia and diabetes insipidus: results in 5 patients. Acta Haematol 93: 1-4, 1995.

5. Nieboer P, Vellenga E, Adriaanse R and van de Loosdrecht AA: Central diabetes insipidus preceding acute myeloid leukemia with $\mathrm{t}(3 ; 12)(\mathrm{q} 26 ; \mathrm{p} 12)$. Neth J Med 56: 45-47, 2000.

6. Wössmann W, Borkhardt A, Gossen R, Göbel FJ and Reiter A Acute myeloid leukemia presenting with diabetes insipidus. Eur J Pediatr 161: 161-162, 2002

7. Harb A, Tan W, Wilding GE, Battiwalla M, Sait SN, Wang ES and Wetzler M: Acute myeloid leukemia and diabetes insipidus with monosomy 7. Cancer Genet Cytogenet 190: 97-100, 2009.

8. Saborio P, Tipton GA and Chan JC: Diabetes insipidus. Pediatr Rev 21: 122-129, 2000.

9. de la Chapelle A and Lahtinen R: Monosomy 7 predisposes to diabetes insipidus in leukaemia and myelodysplastic syndrome. Eur J Haematol 39: 404-411, 1987.

10. Müller CI, Engelhardt M, Laubenberger J, et al: Myelodysplastic syndrome in transformation to acute leukemia presenting with diabetes insipidus: Due to pituitary infiltration association with abnormalities of chromosome 3 and 7. Eur J Haematol 69: 115-119, 2002.

11. Martinelli G, Ottaviani E, Buonamici S, et al: Association of 3q21q26 syndrome with different RPN1/EVI1 fusion transcripts. Haematologica 88: 1221-1228, 2003.

12. Jółkowska J and Witt M: The EVI-1 gene - its role in pathogenesis of human leukemias. Leuk Res 24: 553-558, 2000.

13. Gröschel S, Lugthart S, Schlenk RF, et al: High EVI1 expression predicts outcome in younger adult patients with acute myeloid leukemia and is associated with distinct cytogenetic abnormalities. J Clin Oncol 28: 2101-2107, 2010.
14. Catovsky D, Matutes E, Buccheri V, Shetty V, Hanslip J, Yoshida N and Morilla R: A classification of acute leukaemia for the 1990s. Ann Hematol 62: 16-21, 1991.

15. Montecucco C, Cazzola M and Ascari E: Diabetes insipidus in the preleukaemic phase of acute non-lymphocytic leukaemia. A monosomy 7-associated condition? Scand J Haematol 33: 326-327, 1984.

16. Piccin A, Raimondi R, Laspina S, Marchi M, Rodeghiero F and Rovigatti U: Erythroleukaemia, diabetes insipidus and hypophyseal damage: Two case reports. Leuk Res 31: 1135-1139, 2007.

17. Lavabre-Bertrand T, Bourquard P, Chiesa J, Berthéas MF, Lefort G, Taïb J, Lavabre-Bertrand C, Navarro $M$ and Bureau JP: Diabetes insipidus revealing acute myelogenous leukaemia with a high platelet count, monosomy 7 and abnormalities of chromosome 3: a new entity? Eur J Haematol 66: 66-69, 2001

18. Breccia M, Petti MC, Ottaviani E, Mancini M, D'Elia GM, Mecarocci S and Alimena G: Diabetes insipidus as first manifestation of acute myeloid leukaemia with EVI-1-positive, $3 q 21 q 26$ syndrome and T cell-line antigen expression: what is the EVI-1 gene role? Br J Haematol 118: 438-441, 2002.

19. Nussey SS, Ang VT, Bevan DH and Jenkins JS: Human platelet arginine vasopressin. Clin Endocrinol (Oxf) 24: 427-433, 1986.

20. Dilek I, Uysal A, Demirer T, Koç H, Ozcan M, Keleş H, Numaoğlu N, Ustün C and Ciftçi E: Acute myeloblastic leukemia associated with hyperleukocytosis and diabetes insipidus. Leuk Lymphoma 30: 657-660, 1998.

21. Curley C, Kennedy G, Haughton A, Love A, McCarthy C and Boyd A: Acute myeloid leukemia, the 3q21q26 syndrome and diabetes insipidus: a case presentation. Asia Pac J Clin Oncol 6: $77-79,2010$ 